\title{
An Agent-based Evaluation Model of Students' Emotional Engagement in Classroom
}

\author{
Moamin A. Mahmoud ${ }^{1}$ \\ Institute of Informatics and Computing in Energy (IICE) \\ Universiti Tenaga Nasional \\ Malaysia
}

\author{
Latha Subramainan ${ }^{2}$ \\ College of Graduate Studies \\ Universiti Tenaga Nasional \\ Malaysia
}

\author{
Ihab L Hussein Alsammak ${ }^{3}$ \\ Directorate General of Education of Karbala \\ Ministry of Education \\ Iraq
}

\author{
Mahmood H. Hussein ${ }^{4}$ \\ Faculty of Computer Science and Information Technology \\ University of Malaya \\ Malaysia
}

\begin{abstract}
This study proposes an agent-based evaluation model of students' emotional engagement in a classroom. The proposed model consists of four main elements in a classroom which are, the selected strategy to control engagement, the engagement level of students, the emotional state of a lecturer, and the emotional states of students. The process starts with a lecturer selecting a strategy, which in turn influences the students' emotional state. By utilizing the three variables, students' misbehaviors, motivation, and participation, the engagement level of students is measured that eventually influences the lecturer's emotion either positively or negatively. If negatively, the lecturer proposes another strategy that would trigger the students' emotions and eventually improves the students' engagement level. We simulate our model to validate the applicability and functionality of the model. The simulation result shows a promising application to simulate a classroom environment with very flexible settings that leads to results in less time and cost. It also shows to be widely utilized by researchers in the field of social studies for further investigation of the problem of students' engagement by conducting experiments and report the results.
\end{abstract}

Keywords-Students' emotional engagement; agent-based evaluation; computational model

\section{INTRODUCTION}

Students' engagement in classrooms is a long-standing issue that needs to be objectively and cooperatively resolved or mitigated. It has always been regarded as a crucial factor that influences several educational outcomes [1]. Emotionally engaged students are highly motivated to attend classes, and actively participate in discussions and assessments [2]. On the other hand, emotionally disengaged students are usually habitual truants and/or occasionally misbehave in classrooms [3]. According to Yazzie-Mintz [4], studies have highlighted that boredom is a sign of lack of engagement during a lesson. For instance, bored students may apply less effort and stop paying attention to their lecturer, subsequently becoming even more bored over time and tend to do other unnecessary things. Such students ultimately end up getting poor academic results, involving in many disciplinary actions, and occasionally dropping out of colleges. As expected by the researchers, the study found that teaching practices, teachers' and peers' support, and parental emotional support have a significant relationship with students' engagement. Research studies also reported that educators in many universities use a teachercentered learning process strategy, which lacks personal autonomy, instead of a student-centered strategy [5] [6]. Asian students are notorious for their low-level class participation, it was revealed that less than $20 \%$ of students ask questions during class [7] [8]. These phenomena are due to their disinterest or boredom with ongoing lessons and are not concerned to participate in-class activities. Malaysia PISA survey measured students' engagement and found $80 \%$ of the participating schools fell into the poor performance bracket [9] [10]. Teaching strategies also have been seen as one of the support factors for students' engagement in schools [11].

Several research studies have been conducted to study the problem of poor engagement in classrooms using traditional methods such as questionnaire surveys, experimentation, and analysis. A case study was conducted to examine Malaysian school students' engagement status and to understand the factors that influence students' engagement in three psychological domains (behavior, emotional, and cognitive). Overall, the results show that the level of students' engagement is mostly influenced by emotion, followed by cognitive and behavior [30]. Studies by UCLA Higher Education Research Institute and British universities found that $40 \%$ of students are frequently bored in class due to poor engagement in classrooms [12] and 59\% found lectures are boring in at least half of their classes [13]. Similarly, an annual survey by Indiana University's High School, discovered that about $30 \%$ of the students indicate that they are bored due to the lack of interaction with teachers and $75 \%$ report that the subjects being taught are not interesting [14]. Despite the widespread studies of student engagement in the higher learning institutions of Australia, the USA, and Canada, students' engagement research in Malaysian public universities is scant [15]. However, existing work on student engagement research suffers from the following deficiencies: 
1) Many of the experiments conducted by social science researchers used traditional methods that consume more time and effort. Furthermore, since these experiments are applied to humans, there are very limited settings to be tested and the costs would be excessive if multiple settings are applied [16]. Studies have indicated that it is challenging to manage and control humans to conduct multiple experiments or repeat experiments with the same settings [17]. Thus, traditional methods have a poor success rate in mitigating the problem.

2) Social studies have identified three dimensions of student engagement which are Behavioural Cognitive, and Emotional engagement [18]. While ample research has been conducted on Behavioural and Cognitive engagement, Emotional engagement has received little attention [19]. This is attested by Pekrun et al. [20] who surmises that research on emotion has mostly been neglected as a factor to improve students' engagement although researchers from social studies emphasize the importance of emotion on students' engagement.

3) On the other hand, when it comes to emotional disengagement, a survey of student engagement reported student felt so bored in class, $81 \%$ of the students responded that the material wasn't interesting and $42 \%$ of the students felt that lack of relevance caused boredom [21]. The perceived uninteresting teaching strategy is the biggest contributing factor that needs to be improved upon. However, the development of interesting strategies takes a great deal of time and effort. Besides that, there are tons of strategies that have been proposed by many researchers. Unfortunately, the researchers do not provide the applicability of the strategies in any domain. Moreover, there is no proper centralized library to hold a collection of strategies as the strategies are scattered on the Internet. It is essential to design strategies that help students connect with learning and improve engagement [31] [32] [33].

To overcome these limitations, we propose a study to formulate an agent-based evaluation model of students' emotional engagement in a classroom. In this research, software agents will be empowered to simulate the different states of students' emotions to animate a scenario that reflects or simulates a real classroom environment [27] [28] [29]. To do so, this work primarily focuses on student engagement and its possible classroom teaching strategies to improve students' negative emotional states and engagement performance. We discuss several factors that have been influencing the student engagement process in the literature. However, we emphasize emotions as a factor to shape student engagement as research on emotions in education has received a great deal of attention among educational psychologists [22] [34] [35]. To complete the scope of the engagement, we identify the common emotional experiences of students and lecturer that takes places in a classroom that reflect the engagement level. We then proposed a method to measure the engagement level in a classroom by utilizing three engagement factors [36] [37]. On the other hand, to propose possible strategies to cater to any of the negative emotional states of students, we identify two sets of attributes related to this study, environmental and emotional attributes through the literature. Environmental attributes include a number of students (30 or 60 or 90 or 120), class session (e.g. morning, afternoon/evening), class duration (1 hour or 2 hours or more), type of subject (e.g. theoretical or conceptual), year of study (junior or senior), lecturer style (controlling or autonomy-supportive) and emotional attributes are those such as negative emotional states of students (e.g. anger, anxiety or boredom). Finally, we create a virtual environment simulating classroom dynamics by animating a lecturer agent. The lecturer can insert any strategy using our strategy specification setting and test it in an environment setting to observe the success rate of each strategy to improve student engagement based on their classroom environmental factors. Figure 1 shows the scope of this thesis.

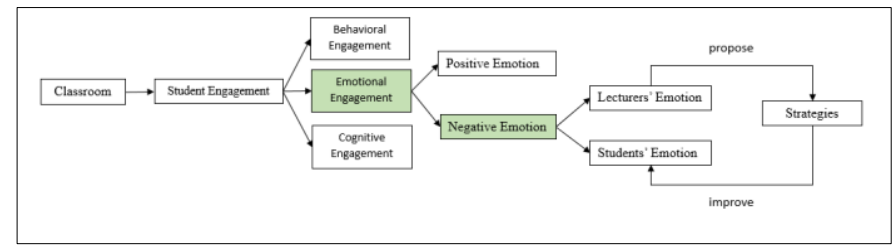

Fig. 1. Research Scope.

The outcomes of this study include an agent-based evaluation model, a method to evaluate emotional engagement level, identify emotional states of students, and propose the most suitable teaching strategies with strategy strength and a classroom dynamics simulator. Researchers and educationists could utilize the simulator to investigate the problem of students' engagement. This could benefit schools and universities by giving teachers and lecturers exposure to students' engagement for improved academic performance.

\section{Agent-Based Evaluation Model}

Typically, a lecturer monitor student's engagement level in a classroom via indirect indicators, for instance, amount of participation in classroom discussion, attendance, commitment on a task given, time spent on assessments, the intensity of concentration during the ongoing lesson, and amount of motivation or interest shown on particular course material Lamborn, (1992). The preliminary model of this study is based on four main elements in a classroom; the selected strategy to control engagement, the engagement level of students, the emotional state of students, and lecturer emotion. An applied strategy during a classroom session influences the students' engagement-based emotions. By utilizing three variables, students' misbehavior, poor motivation, and poor participation, the engagement level of students can be measured. Student's emotional state can be identified through analysis of engagement level. A negative student's emotional state and the result of engagement measurement trigger the lecturer's emotion either positively or negatively. If affected negatively, the lecturer proposes another strategy that would trigger students' emotions positively and eventually improve engagement. The lecturer again measures the engagement level and decides whether to take another strategy or maintain the existing one. Figure 2 illustrates the agent-based evaluation model. 


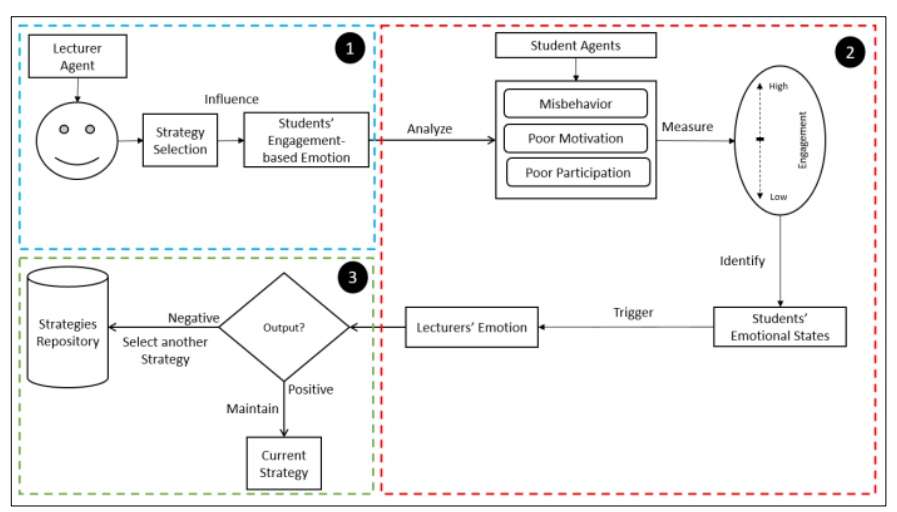

Fig. 2. Agent-based Evaluation Model.

As shown in Figure 2, the classroom simulator model is divided into three main components. The first component shows the Lecturer Agent (AL) Components that involve a strategy element. The AL applies the strategy during the classroom session. The effectiveness of the strategy applied influences students' engagement-based emotions. The second component shows the Engagement Measurement by analyzing the factors of misbehavior, poor motivation, and participation. Students' emotional state can be predicted through the result of engagement. Thus, negative emotional states of students and the level of engagement affect the AL emotions which trigger either positively or negatively. The third component shows the Strategy Revision where the negative emotion of the lecturer triggers the AL to select a new strategy that would improve their engagement in the classroom. The AL maintains the current strategy if positive emotion occurred. The process continues until the student displays positive factors of behavior, a high level of motivation, and participation. The logical model has been presented in each component in the next section.

\section{A. First Component - Lecturer Agent and Strategy Selection}

1) Modelling lecturer agents' architecture: In the BeliefDesire-Intention (BDI) architecture [26], agents are built with mentalistic notions to recognize or deliberate their goals. An agent's belief represents its knowledge about the environment. It forms its belief from the state of the environment. If there are changes in the state of the environment, it updates its belief, which changes its behavior that leads to the achievement of its goal. The Emotional component represents the emotions being experienced by the lecturer towards students' engagement in a classroom medium, high, or very high influenced by strategy application. The Belief component is associated with the Desire component aims to achieve an acceptable engagement level by revising or maintain a strategy determined by the Intentional component. These emotions trigger the Lecturer's Belief about the current engagement level that is either very low or low. The agent belief will be triggered when the LA receives or observes a negative signal(s) of students' engagement. When the Belief is triggered (e.g. feels worried or upset), the LA Desire will be revising the current strategy based on the received emotion to enhance the engagement. Once the LA figured out a better strategy, the intention will be to implement the revised strategy.

2) Strategy selection: It begins with strategy selection by the lecturer. As explained earlier, a few factors have been influencing classroom strategy. In our study, we only emphasize class environment factors such as the number of students (30 or 60 or 90), class session (eg. morning, afternoon/evening), class duration (1hour or 2 hours or more), type of subject (e.g. theoretical or conceptual), and year of the student (junior or senior). Once the lecturer entered the environmental setting data, the next step is to evaluate student's behavior (engagement data) based on previous classroom sessions and finally select a strategy from the dropdown list. The effectiveness of any strategy applied in turn influences students' engagement-based emotion.

3) Logical model of strategy selection: Once the lecturer selects a strategy and runs the environment setting, the AL capture the environment setting data and test the effectiveness of the selected strategy. AL analyses the data inserted and produce the strength of the selected strategy in percentages. Besides that, AL can cross-find the nearest matched strategies with environment and engagement data entered by the lecturer. Figure 3 shows the strategy, selection model. We define the terms Environment Factors and Engagement Factors as follows:

Definition 1: Environmental factors, EnvFact consist of Number of Students, Class Session, Class Duration, Type of Subject, and Year of Study.

Definition 2: Engagement factors, EngmtFact constitute of Misbehavior, MisB, Poor Motivation, Mtv, and Poor Participation, Ptc.

Definition 3: A Strategy Selection, StrgySelect constitutes of Environmental Factors and Engagement Factors.

StrgySelect:\{ EnvFact, EngmtFact \}

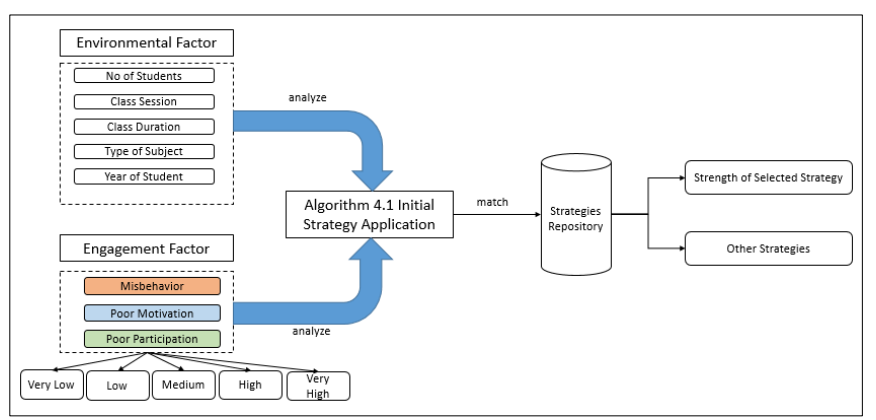

Fig. 3. Strategy Selection Model.

There are various classroom environmental factors have to be considered before a lecturer or social studies researchers can identify the best teaching strategy to be applied to keep student remain engaged during the lesson. For example, they need to consider the number of students, a class session with the duration of the lesson, the type of subject to be taught, and the seniority of students. The way students behaving, level of motivation, and amount of participation during a lesson reflects 
how much they are engaged with the lesson. By considering these factors, a lecturer would have a clear idea of a suitable teaching strategy to be applied. Using this tool, the lecturer can find out the strength of the selected teaching strategy according to the environmental and engagement factors. However, if he/she made a poor selection teaching strategy, the agent proposes the best three strategies.

\section{B. Second Component - Engagement Measurement}

As explained in Section 1, the most prominent emotional states that occurred in a classroom include enjoyment, pride, anger, anxiety, and boredom. For example, before a classroom lesson start, less prepared or low self-efficacious students are more likely to experience negative emotions. In our context, anger, anxiety, and boredom represent the states of negative emotions. In contrast, well-prepared or highly self-efficacious students are evaluated to be in a state of positive emotional state. Enjoyment and pride emotions represent the elicited positive emotional state for our context at the beginning of the lesson. By comparison, students are expected to experience different elicited emotional state after the lecturer change their teaching strategy. At this stage, different learning strategies give rise to elicited emotions. For example, a desirable strategy (e.g. engaged in peer review) elicits a state of positive emotions. In contrast, an undesirable strategy (e.g. reading slide contents) elicits a state of negative emotions. However, literature reported that a lecturer needs to change their teaching strategies only when they observed the students are experiencing negative emotional states. To evaluate the student's emotional state, we need to identify the values of the engagement factors (e.g. Misbehaviour, Poor Motivation, and Poor Participation) that have been influencing the engagement level in a classroom and subsequently the lecturer's emotions. These are the potential factors to infer students' engagement levels. Generally, negative emotional states will impact engagement level, however, we need to identify the intensity of negative emotions influencing the students in a classroom. This is because several emotions can occur at the same time among different individuals. Changes in the emotional states of students consequently reflect the engagement level in a classroom. Therefore, several student behavior indications reveal the emotional states of students. It is a well-known fact from many studies that indicates a positive emotional state leads to high-level engagement in the classroom including no misbehaving attitude from a student, a high level of motivation, and good participation in class discussion. Whereas, negative emotional states will lead to low engagement whereby students pay no attention and started to do their work with no motivation and participation.

1) Engagement level: In the past, many researcher proposed techniques to measure the engagement, and its' have been practiced in a different context to identify if students are actively engaged in the learning process. These measures emphasize the traditionally "quantifiable" aspects of attendance rates, truancy, time-on-task, and consequently suspension/discipline rates [23]. Besides quantifiable methods, self-report, teacher ratings, interviews, observations, crosscultural data, and assessment grades also have been in practice. Despite the traditional method of measuring engagement, researchers stressed that more systematic and thoughtful attention to the measurement of engagement is the most imperative direction for future research. [24].

Therefore, in our study, we anticipate modeling how student engagement can be measured based on the variable of engagement factors; misbehavior, motivation, and participation of students. We use six-level scales; not present, very low, low, medium, high, and very high to rate student behaviors from various engagement factors parameters. To determine the current engagement level, the lecturer is required to rate student behavior in percentages based on their interaction with their student in the last class session. Therefore, they are required to use six-level scales as shown in Table 1 to determine the student behaviors. In this section, we use the ordinal scale by Stevens (1946) to determine the engagement level as follows in Table 1.

The process starts with measuring the engagement level of students by the lecturer via indicators of three variables: students' misbehaviors, poor motivation, and poor participation. We have a total of 11 indicators for the 3 engagement factors (eg. Misbehavior (4 indicators), Poor Motivation (4 indicators), and Poor Participation (3 indicators). Once we analyzed the value of engagement factors, we can identify the current engagement level in a classroom. To measure the engagement level of students from various factors, we use the ordinal scale as per Table 1. Figure 4 shows the engagement level analysis model. We define the terms Engagement Level as follows:

Definition 4: Engagement level, EngLvl can be measured based on average values of engagement factors; MisB, Mtv, and Ptc level in the classroom.

2) Modeling student agents: The students' agents in this classroom simulator tools are very basic. This agent reflects the behavior as indicated by the academician. For example, let's denote the number of a student as agent $\alpha$, misbehavior indicators as $\mathrm{Xn}$, poor motivation indicators as $\mathrm{Yn}$, poor participation as $\mathrm{Zn}$, and rating values as values (e.g. 0 if not present and 1 if present, if the rating falls into any level of scales), Table 2 shows the indicators of each behavior.

We use the randomize function to randomly assign values to student agents. For instance, student behavior $(\alpha 1, \mathrm{X} 3,1)$. Table 3 shows an example of evaluating student behavior for a class of ten students.

TABLE I. SIX LEVEL SCALES

\begin{tabular}{|c|c|c|c|c|c|}
\hline $\begin{array}{l}\text { Not } \\
\text { Present }\end{array}$ & $\begin{array}{l}\text { Very } \\
\text { Low }\end{array}$ & Low & Medium & High & Very High \\
\hline 0 & $1-20$ & $21-40$ & $41-60$ & $61-80$ & $81-100$ \\
\hline \multicolumn{2}{|c|}{ Engagement Factor } & Very Low & & & \\
\hline \multicolumn{2}{|c|}{ Misbehavior } & Low & & & \\
\hline \multicolumn{2}{|c|}{ Poor Motivation } & & $\begin{array}{c}\text { Algorithm } 4.2 \\
\text { Engagement Level }\end{array}$ & \multicolumn{2}{|c|}{$\rightarrow$ Current Engagement Level } \\
\hline \multicolumn{2}{|c|}{ Poor Participation } & High & & & \\
\hline & & $\begin{array}{l}\text { Very } \\
\text { High } \\
\end{array}$ & & & \\
\hline
\end{tabular}

Fig. 4. Engagement Level Analysis Model. 
TABLE II. INDICATORS OF STUDENTS'BEHAVIOR

\begin{tabular}{|l|l|l|}
\hline \multirow{4}{*}{$\begin{array}{l}\text { Four indicators describe } \\
\text { Misbehaving }\end{array}$} & Verbally or physically aggressive & $\mathrm{X} 1$ \\
\cline { 2 - 3 } & Spoken in a raised voice & $\mathrm{X} 2$ \\
\cline { 2 - 3 } & Acting in an abusive manner & $\mathrm{X} 3$ \\
\cline { 2 - 3 } & Yelling and screaming & $\mathrm{X} 4$ \\
\hline \multirow{4}{*}{$\begin{array}{l}\text { Four indicators describe } \\
\text { poor Motivation }\end{array}$} & Non-attentiveness & $\mathrm{Y} 1$ \\
\cline { 2 - 3 } & Talking out of turns & $\mathrm{Y} 2$ \\
\cline { 2 - 3 } & Playing video games & $\mathrm{Y} 3$ \\
\cline { 2 - 3 } $\begin{array}{l}\text { Three indicators describe } \\
\text { the poor participation }\end{array}$ & Get away from class & $\mathrm{Y} 4$ \\
\cline { 2 - 3 } & Avoiding eye contact & $\mathrm{Z} 1$ \\
\cline { 2 - 3 } & Anability to initiate conversations & $\mathrm{Z} 2$ \\
\hline
\end{tabular}

TABLE III. EXAMPLE OF EVALUATING STUDENT BEHAVIOR

\begin{tabular}{|c|c|c|c|c|c|c|c|c|c|c|c|c|}
\hline & $\bar{x}$ & $x^{2}$ & $x^{2}$ & $x^{ \pm}$ & $\approx$ & $\nu^{0}$ & 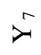 & $\nu^{\infty}$ & $\hat{N}$ & $\stackrel{ }{N}$ & $\bar{N}$ & \\
\hline & \multicolumn{4}{|c|}{ Misbehavior } & \multicolumn{4}{|c|}{ Poor Motivation } & \multicolumn{3}{|c|}{$\begin{array}{l}\text { Poor } \\
\text { Participation }\end{array}$} & \\
\hline$\alpha_{1}$ & 0 & 0 & 0 & 0 & 0 & 0 & 0 & 0 & 0 & 0 & 0 & - \\
\hline$\alpha_{2}$ & 1 & 0 & 0 & 0 & 1 & 0 & 0 & 0 & 0 & 1 & 0 & $\sqrt{ }$ \\
\hline$\alpha_{3}$ & 0 & 1 & 0 & 0 & 0 & 0 & 0 & 0 & 0 & 0 & 0 & $\sqrt{ }$ \\
\hline$\alpha_{4}$ & 1 & 0 & 0 & 0 & 0 & 0 & 0 & 1 & 0 & 0 & 1 & $\sqrt{ }$ \\
\hline$\alpha_{5}$ & 0 & 0 & 1 & 0 & 0 & 1 & 0 & 0 & 1 & 0 & 0 & $\sqrt{ }$ \\
\hline$\alpha_{6}$ & 0 & 0 & 0 & 1 & 1 & 0 & 0 & 0 & 0 & 0 & 0 & $\sqrt{ }$ \\
\hline$\alpha_{7}$ & 0 & 1 & 0 & 0 & 0 & 0 & 0 & 0 & 0 & 1 & 0 & $\sqrt{ }$ \\
\hline$\alpha_{8}$ & 1 & 0 & 0 & 0 & 1 & 1 & 0 & 0 & 0 & 0 & 0 & $\sqrt{ }$ \\
\hline$\alpha_{9}$ & 0 & 0 & 0 & 0 & 0 & 0 & 0 & 0 & 0 & 0 & 0 & - \\
\hline \multirow[t]{2}{*}{$\alpha_{10}$} & 0 & 0 & 0 & 0 & 0 & 0 & 0 & 0 & 0 & 0 & 0 & - \\
\hline & \multicolumn{4}{|l|}{7} & \multicolumn{4}{|l|}{5} & \multicolumn{3}{|l|}{4} & \\
\hline \multicolumn{12}{|c|}{ Total number of students who are not engaged to lesson } & 7 \\
\hline
\end{tabular}

By analyzing Table 3, 7 out of 10 students are not engaged in the lesson, only 3 of them are engaged in the lesson. Therefore, current Engagement Level is (3/10) $* 100=30 \%$. According to our ordinal scales in Table 1, the current engagement level is LOW. However, the total number of students in the classroom has an impact on the result of the engagement level. This is because students experience more than one type of emotional state at one time.

3) Identify the emotional states of student: The $\mathrm{AL}$ can identify the emotional states of students based on the values of engagement factors (refer to Table 3). In this section, we use the ordinal scale by Hogan and Warrenfeltz [25] to determine the scale of the outcomes for each factor in Table 2. Once we analyzed the value of engagement factors, we can identify the intensity of the emotional states of students. Figure 5 shows the identification of the intensity of the emotional state's model. However, to conclude the values of the emotional factors, we use the ordinal scale by Hogan and Warrenfeltz [25] to determine the scale of the outcomes for each factor in Table 4.
TABLE IV. ORDINAL SCALE [25]

\begin{tabular}{|l|l|l|}
\hline LOW & MEDIUM & HIGH \\
\hline $10 \%-39 \%$ & $40 \%-69 \%$ & $70 \%-100 \%$ \\
\hline
\end{tabular}

We define the terms Emotional Factors as follows:

Definition 5: Emotional factors, EmoFact constitute of Anger, Boredom, and Anxiety derived from EngmtFact.

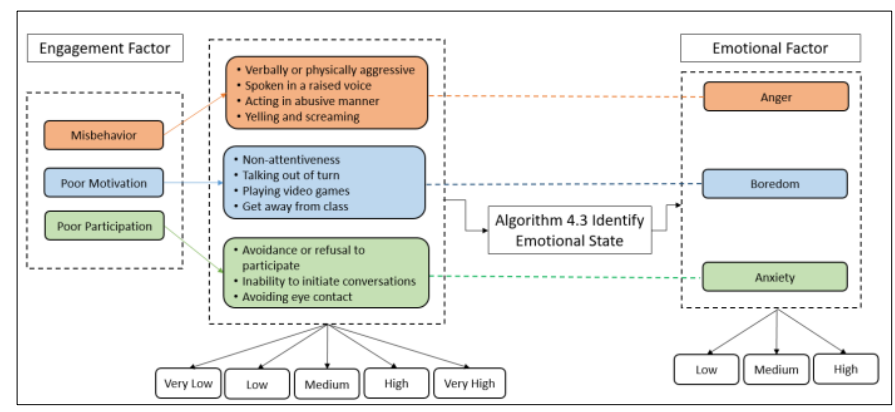

Fig. 5. Identification of Intensity of Students' Emotional States Model.

Therefore, according to Table 4

a) Total number of Students' Misbehavior is 7, therefore (7 / 10) $* 100=70 \%$ - High.

b) Total number of Students' Poor Motivation is 5, therefore $(5 / 10) * 100=50 \%$ Medium.

c) Total number of Students' Poor Participation is 4, therefore $(4 / 10) * 100=40 \%$ Medium.

As a result, the intensity of the emotional states of students as follows:

EmoFact $($ MisB $)=($ Anger, High $)$

EmoFact $($ Mtv $)=($ Boredom, Medium $)$

EmoFact $($ Ptc $)=($ Anxiety, Medium $)$

The lecturer measures the engagement level of students by rating the students' behaviors based on past experiences. From engagement factors result, The agent can derive the intensity (eg. low, medium, high) of emotional states of students; anger, boredom, and anxiety.

4) Identify lecturer emotion: Lecturer's emotions are as important as students' emotions. The result of the engagement measurement positively or negatively affects a lecturer's emotion. If negatively, the lecturer deploys another strategy that triggers positive students' emotions of which would eventually improve engagement. In contrast, the positively affected teacher is expected to engage a strategy that would maintain a positive aura to his students. According to literature, student emotional state also impacts on lecturer emotion. This engagement assessment cycle should continue to maintain positivity throughout the students learning sessions. Figure 6 shows the lecturer's emotion model. We define the terms Lecturer emotion as follows:

Definition 6: Lecturer emotion, EmLec is being affected by the emotional state of student Emotional Factor, EmoFact, and Engagement Level, EngLevel. 


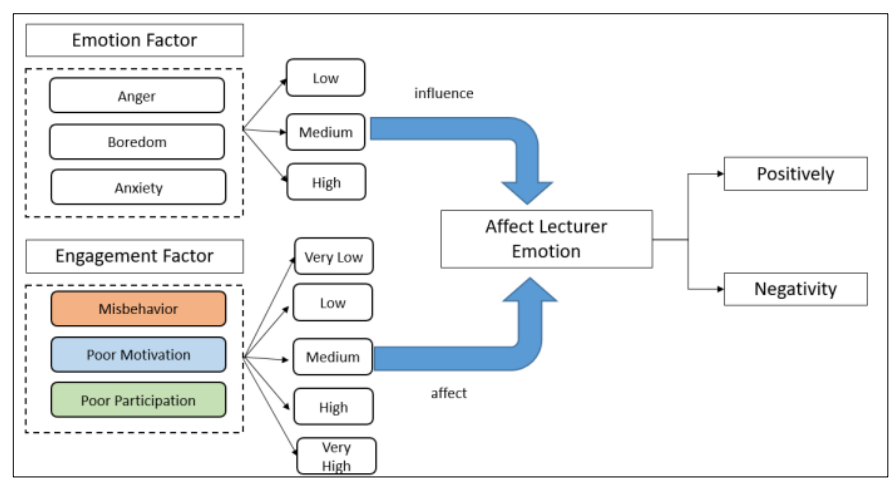

Fig. 6. Lecturer Emotion Model.

A lecturer is always concern about the engagement level of students during a lesson that directly impacts their emotions. It impacts the lecturer's emotions either positively or negatively. For example, low engagement levels affect the emotion of the lecturer negatively and vice versa. If she/he is affected negatively, it would trigger them to change their teaching strategy to attain back their students' attention. However, in this thesis, we focus formulation of identifying students' emotional states only. Lecturer emotions are stated in the agent-based evaluation model to complete the whole cycle of enhancing the student engagement process. As per in the literature, students' emotional state equally impacts the lecturer's emotions either positively or negatively that eventually triggers the lecturer to deploy another teaching strategy to keep students remain engaged with the lesson. Therefore, we do not have any formulation to identify lecturer emotions in this classroom simulator tool as we focus on identifying the students' emotional states. Moreover, the lecturer agent can measure the engagement and identify the emotional states of students however identify or improving lecturer emotions is not our scope of the study.

\section{Third Component - Strategy Revision}

Once the lecture emotion is being affected negatively, the lecturer can implement other strategies as been proposed by the $\mathrm{AL}$, then test the effectiveness of the new strategy again. Besides that, a lecturer, who might have ideas on new strategies based on their teaching experience, can insert a new strategy using the Strategy Specification Settings Interface and use the Likert scale to appropriately assign the impact of the strategy. The interface consists of a new strategy text field, a number of students (30 or 60 or 90), class session (eg. morning, afternoon/evening), class duration (1hour or 2 hours or more), type of subject (eg. theoretical or conceptual), and years of a student (junior or senior) in the drop-down list. For example, a lecturer from the Department of software engineering would like to register a new strategy that he had tried in his classroom and found he can improve the engagement level from low to high. However, he needs to indicate the class environment factors that are influence his success rate of strategy. For instance, his strategy for software testing theory subject only works for 60 third-year students, with a 2-hour duration at the morning session. He also needs to indicate the intensity of emotional states that he plans to improve (e.g high, medium, and low). Figure 7 shows the new strategy storing model. We define the NewStrategy Application terms as follows:

Definition 7: NewStrategy application to be stored in Strategy Repository, StrgyRepo constitutes of Environmental Factors, EnvFact and Emotional Factors, EmoFact.

Once the lecturer filled up all the information requested, the strategy will be are stored in a repository as text files. Each new strategy application will be saved as a file. Later on, if the lecturer intends to edit the strategy, he/she can do so.

1) Proposing best three strategies: In the environment specification setting, it begins with strategy selection where the lecturer needs to key in information of her/his class environment and estimates the percentage of student behaviors based on their last class session experience which eventually predicts the students' emotional states and engagement level. Then, they are required to select a strategy from the list. As output, the AL will display the engagement level and emotional states of students. The strength of the selected strategy in percentage will be displayed too. Besides, the agent can propose other strategies based on the environmental and engagement data inserted by the lecturer. Meanwhile, in a strategy specification setting, a lecturer can store new strategies that include environmental and emotional factors. Therefore, using two settings information, the AL can propose the best three strategies that suit the environment setting data entered by the lecturer. We define the terms environment setting and strategy specification setting as follows:

Definition 8: Environment Setting, EnvSet comprises of EnvFact, EngmFact, EmoFact, EngLevel, StrgySelect, and EmLec. Strategy Specification Setting, StrgySpecSet comprises of StrgyRepo.

Definition 9: Strategies Strength, Strgy Strength comprises EnvSet and StrgySpecSet

\section{Strgyn Strength: $\{$ EnvSet $\cap$ StrgySpecSet $\}$}

Therefore to propose best 3 strategies:

Strategy Selection: MAX (Strgy1 Strength, Strgy2 Strength, Strgy3 Strength... n++).

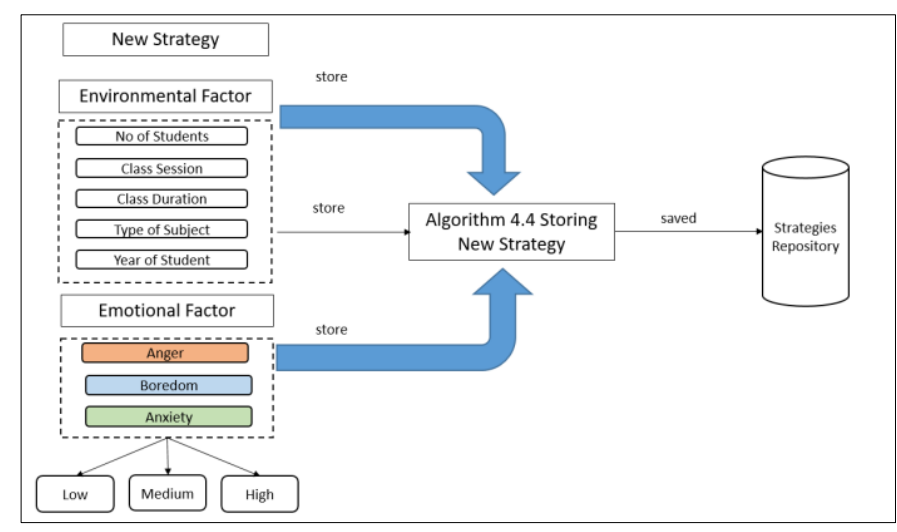

Fig. 7. New Strategy Storing Model. 
However, to find the best 3 strategies' effectiveness, we need to analyze the Strgy Strength data. We have a total of 8 factors as follows:

a) Environmental Factors (5) - Number of Students, Class Session, Class Duration, Type of Subject, and Year of Study.

\section{Anxiety.}

b) Emotional Factors (3) - Anger, Boredom, and

Hence, we will find the average of each scenario then divide it by 8 then convert it into percentages.

In this case scenario, the lecturer chose the Online Quiz strategy for his 2 hours, morning session class with 30 senior students, for his Software Quality theoretical subject. However, according to our simulator, the Online Quiz strategy does not apply to his environmental setting as the strategy strength is $12.5 \%$. In other words, deploying this online quiz strategy for his class situation will lead to low engagement as students already experiencing medium-level anger and boredom, and high anxiety. This emotional feedback revealed as some of the students might not ready for an online quiz as no early preparation was done and some might be bored to answer questions so they might not pay attention to the questions. Therefore, the AL can propose other better strategies suitable for his environmental setting. In this case, Peer Review is the most suitable strategy to be applied for morning session classes for senior students as it has $87.5 \%$ strength followed by applying Real Life Scenarios strategy which is $62.5 \%$, and Discovery and Discussion strength is $50 \%$.In conclusion, by deploying a Peer Review strategy, the academician can cater to medium-level anger, and a high level of boredom and anxiety students and thus improve their emotional states and engagement level.

\section{Developing the Agent-Based Evaluation Model USING PROLOG}

We have chosen Prolog to simulate an agent-based evaluation model to create a real-world implementation for the classroom domain. We use Win-Prolog and its extended module Chimera, which can handle multi-agent systems. We use Prolog for two reasons: firstly, Prolog is well suited for expressing complex ideas because it focuses on the computation's logic rather than its mechanics where the drudgery of memory allocation, stack pointers, and computational engine. Secondly, since Prolog incorporates a logical inferencing mechanism, this powerful property can be exploited to develop inference engines specific to a particular domain.

\section{A. Process Flow of Classroom Simulator Tool}

The simulator is divided into two main settings which are Environment Specification Setting and Strategy Specification Setting. The main use for these two main settings is the academician and social studies researchers. In Environment Setting, the academician or the researcher can test the strategy selected whereas in Strategy Specification Setting, the lecturer can store their ideas of strategy, and then they can test it on Environment Setting to observe how much it can improve the student engagement performance in a classroom. Humans as Lecturer agents (AL) communicate with their agents via an interface and the agents monitor and update their environment to communicate between agents, perform tasks that enable the progression of the workflow. For an initial strategy application, it constitutes environmental factors and engagement factors. From the engagement factors, our AL can derive the engagement level (e.g. very low, low, medium, high, very high) and emotional factors which include the negative emotional states of the student (e.g. anger, anxiety, or boredom).

A teaching strategy is applied based on a set of environment and engagement data, AL will analyze the data inserted by the lecturer. AL then measures the engagement level in the classroom by analyzing the engagement factors and emotional state of students can be predicted. In this case point, the ineffectiveness of a strategy can cause boredom among students thus will lead to low engagement. Subsequently, low engagement and negative emotional state of students affect lecturers' emotions. If affected positively, the lecturer can maintain the strategy, otherwise, implement another most suitable strategy. AL can propose other better strategies. In this simulator, a lecturer, who might have ideas on new strategies based on their teaching experience can store a new strategy application into Strategies Repository constitutes of Environmental Factors and Emotional Factors using a proposed New Strategy Specification Settings Interface. The core modules making up the agent-based evaluation model are the selected strategy to control engagement, the engagement level of students, and the emotional states of students in a classroom. Figure 8 shows the process flow of the classroom simulator tool.

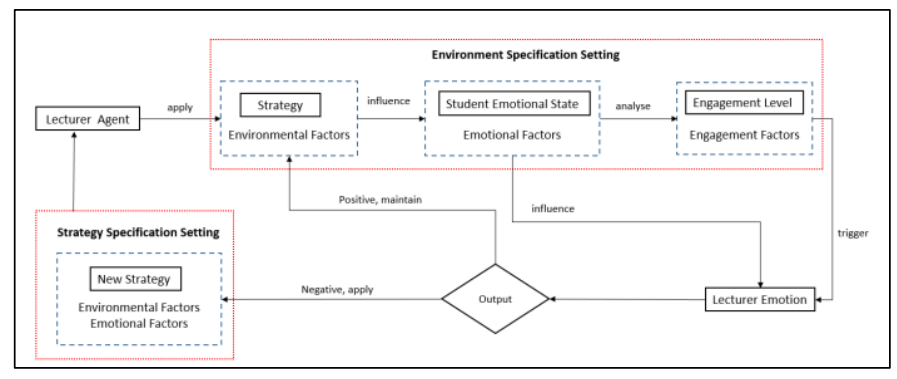

Fig. 8. Process Flow of Classroom Simulator.

\section{B. Scenario-Based Testing}

This section will be divided into two. In the first part, Environmental testing is conducted and in the second part, Strategy Specification testing is conducted. We conduct four scenario-based testing and two new strategies testing to validate our classroom simulator tool to find out the strategy strength and ability of agents to propose the three best strategies according to the environmental setting factors.

\section{1) Environmental setting testing}

a) Scenario A: Lecturer A would like to deploy a strategy for the "Software Testing" theory-based subject which is scheduled on Monday morning 2 hours class session which consists of 90 senior students. the rates of student behavior are assumed as follows in Table 5.

From the analysis of results in scenario $\mathrm{A}$ in Table 5, it shows that engagement level was very low in past class 
sessions. Students experiencing high boredom followed by medium level anger and anxiety. In short, students' poor motivation is a high and medium state of misbehavior and participation among students. Selected strategy result and best 3 strategies proposed by agents as follows in Figure 9.

Analyses of results show that the initial strategy selection which is Discovery and Discussion accordance with the classroom environment is only 50\% applicable. Agent proposed Peer Review would be a better strategy to deploy to improve poor engagement and emotional states of students in scenario A as the strength is $75 \%$ which is higher than Discovery and Discussion strategy strength followed by Real Life Scenario which is $62.5 \%$.

b) Scenario B: Lecturer B would like to deploy a strategy for the "Discrete Structure" practical-based subject which is scheduled on Friday afternoon 2 hours class session which consists of 60 junior students. Based on his last class session, he rates student behavior as follows in Table 6 .

From the analysis of the result in Table 7 accordance with scenario B in Table 7, shows that engagement level was very low in past class sessions. Students experiencing a high level of boredom and anxiety meanwhile anger emotional states do not present. In short, students' poor motivation and participation are high among students. Selected strategy result and best 3 strategies proposed by agents as follows in Figure 10.

TABLE V. RATING STUDENT BEHAVIOR FOR SCENARIO A

\begin{tabular}{|l|l|l|}
\hline Engagement Factor & Indicators & Rating \\
\hline \multirow{4}{*}{ Misbehavior } & Verbally or physically aggressive & Low \\
\cline { 2 - 3 } & Spoken in a raised voice & High \\
\cline { 2 - 3 } & Acting in an abusive manner & Not Present \\
\cline { 2 - 3 } & Yelling and screaming & Not Present \\
\hline \multirow{4}{*}{ Poor motivation } & Non-attentiveness & Very High \\
\cline { 2 - 3 } & Talking out of turns & High \\
\cline { 2 - 3 } & Playing video games & Not Present \\
\cline { 2 - 3 } & Get away from class & High \\
\hline & Avoiding eye contact & Medium \\
\cline { 2 - 3 } & Inability to initiate conversations & Medium \\
\cline { 2 - 3 } & Avoidance or refusal to participate & High \\
\hline
\end{tabular}

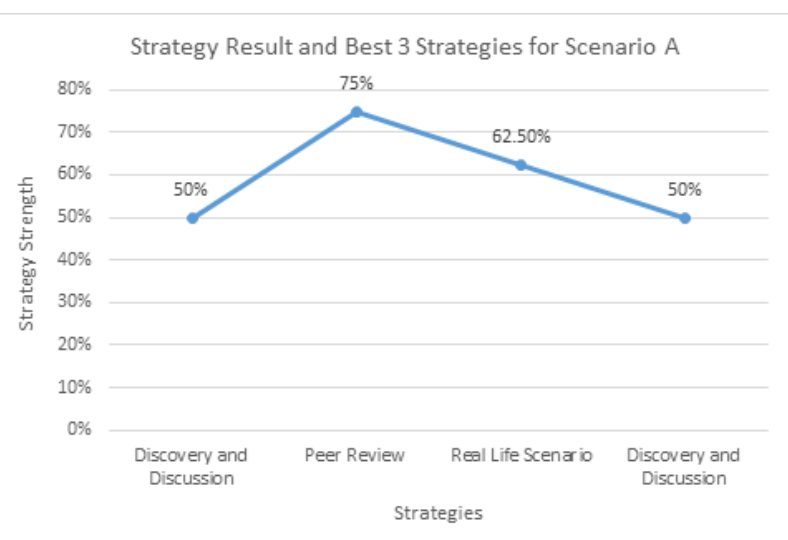

Fig. 9. Selected Strategy Result and Best 3 Strategies for Scenario A.
TABLE VI. RATING STUDENT BEHAVIOR FOR SCENARIO B

\begin{tabular}{|l|l|l|}
\hline Engagement Factor & Indicators & Rating \\
\hline \multirow{4}{*}{ Misbehavior } & Verbally or physically aggressive & Not Present \\
\cline { 2 - 3 } & Spoken in a raised voice & Not Present \\
\cline { 2 - 3 } & Acting in an abusive manner & Not Present \\
\cline { 2 - 3 } & Yelling and screaming & Not Present \\
\hline \multirow{4}{*}{ Poor motivation } & Non-attentiveness & High \\
\cline { 2 - 3 } & Talking out of turns & Medium \\
\cline { 2 - 3 } & Playing video games & Low \\
\cline { 2 - 3 } & Get away from class & Low \\
\hline & Avoiding eye contact & High \\
\cline { 2 - 3 } & Inability to initiate conversations & Low \\
\cline { 2 - 3 } & Avoidance or refusal to participate & High \\
\hline
\end{tabular}

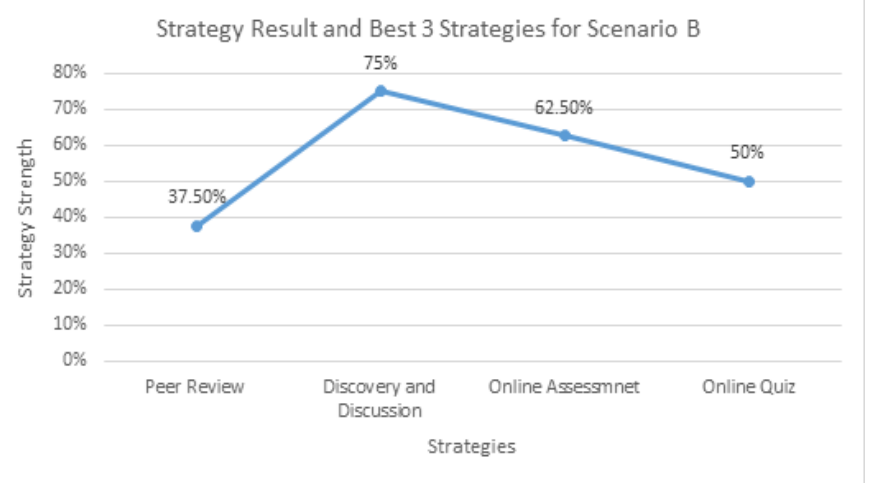

Fig. 10. Selected Strategy Result and Best 3 Strategies for Scenario B.

Analyses of results show that the initial strategy selection which is Peer Review in accordance to the classroom environment is only $37.5 \%$ which is not suitable to deploy. Agent proposed Discovery and Discussion would be a better strategy to deploy to improve poor engagement and emotional states of students in scenario B as the strength is $75 \%$ which is higher than Peer Review strategy strength followed by Real Life Scenario which is $62.5 \%$ and Online Quiz which is $50 \%$.

TABLE VII. RATING STUDENT BEHAVIOR FOR SCENARIO C

\begin{tabular}{|l|l|l|}
\hline Engagement Factor & Indicators & Rating \\
\hline \multirow{4}{*}{ Misbehavior } & Verbally or physically aggressive & Very Low \\
\cline { 2 - 3 } & Spoken in a raised voice & Not present \\
\cline { 2 - 3 } & Acting in an abusive manner & Not present \\
\cline { 2 - 3 } & Yelling and screaming & Not Present \\
\hline \multirow{4}{*}{ Poor motivation } & Non-attentiveness & Medium \\
\cline { 2 - 3 } & Talking out of turns & High \\
\cline { 2 - 3 } & Playing video games & Medium \\
\cline { 2 - 3 } & Get away from class & Medium \\
\hline & Avoiding eye contact & Medium \\
\cline { 2 - 3 } & Inability to initiate conversations & Low \\
\cline { 2 - 3 } & Avoidance or refusal to participate & Medium \\
\hline
\end{tabular}


c) Scenario C: Lecturer C would like to deploy a strategy for the "Operating System Concepts" theory-based subject which is scheduled on Thursday morning 1-hour class session which consists of 30 senior students. Based on his last class session, he rates student behavior as follows in Table 7 .

The analysis of results in Table 6 , it shows that the engagement level was low in past class sessions. Students experiencing a high level of boredom, followed by a medium level of anxiety and a low level of anger. In short, students' poor motivation is a high and medium state of poor participation and a low level of misbehaving among students. Selected strategy result and best 3 strategies proposed by agents as follows in Figure 11.

Analyses of results show that the initial strategy selection which is Online Quiz accordance with the classroom environment is only 50\% applicable. Agent proposed both Peer Review and Online Quiz strategies are the best strategies to deploy to improve the poor engagement and emotional states of students in scenario $\mathrm{C}$ as the strength is $50 \%$ followed by Online Assessment which is only $37.5 \%$. For this scenario C, better strategies need to be stored in a tool that has higher strength than both Peer Review and Online Quiz strength.

d) Scenario D: Lecturer D would like to deploy a strategy for the "Java Programming" practical-based subject which is scheduled on Tuesday afternoon 2-hour class session which consists of 120 senior students. Based on his last class session, he rates student behavior as follows in Table 8 .

The analysis of the result shows that the engagement level was medium in the last class session. Students experiencing a medium level of anger, boredom, and anxiety. In short, a medium state of students' misbehavior, poor motivation, and participation among students. Selected strategy result and best 3 strategies proposed by agents as follows in Figure 12 .

Analyses of results show that the initial strategy selection which is Peer Review in accordance to the classroom environment is only $37.5 \%$ applicable which is not suitable to deploy. Agent proposed Real Life Scenario would be a better strategy to deploy to improve poor engagement and emotional states of students in scenario D as the strength is $62.5 \%$ which is higher than Peer Review strategy strength followed by Online Quiz and Online Assessment which has the same strength as $50 \%$.

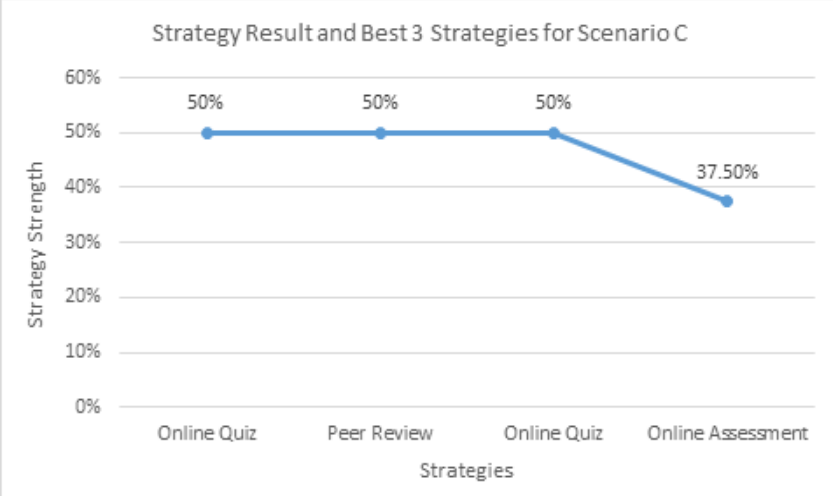

Fig. 11. Selected Strategy Result and Best 3 Strategies for Scenario C.
TABLE VIII. RATING STUDENT BEHAVIOR FOR SCENARIO D

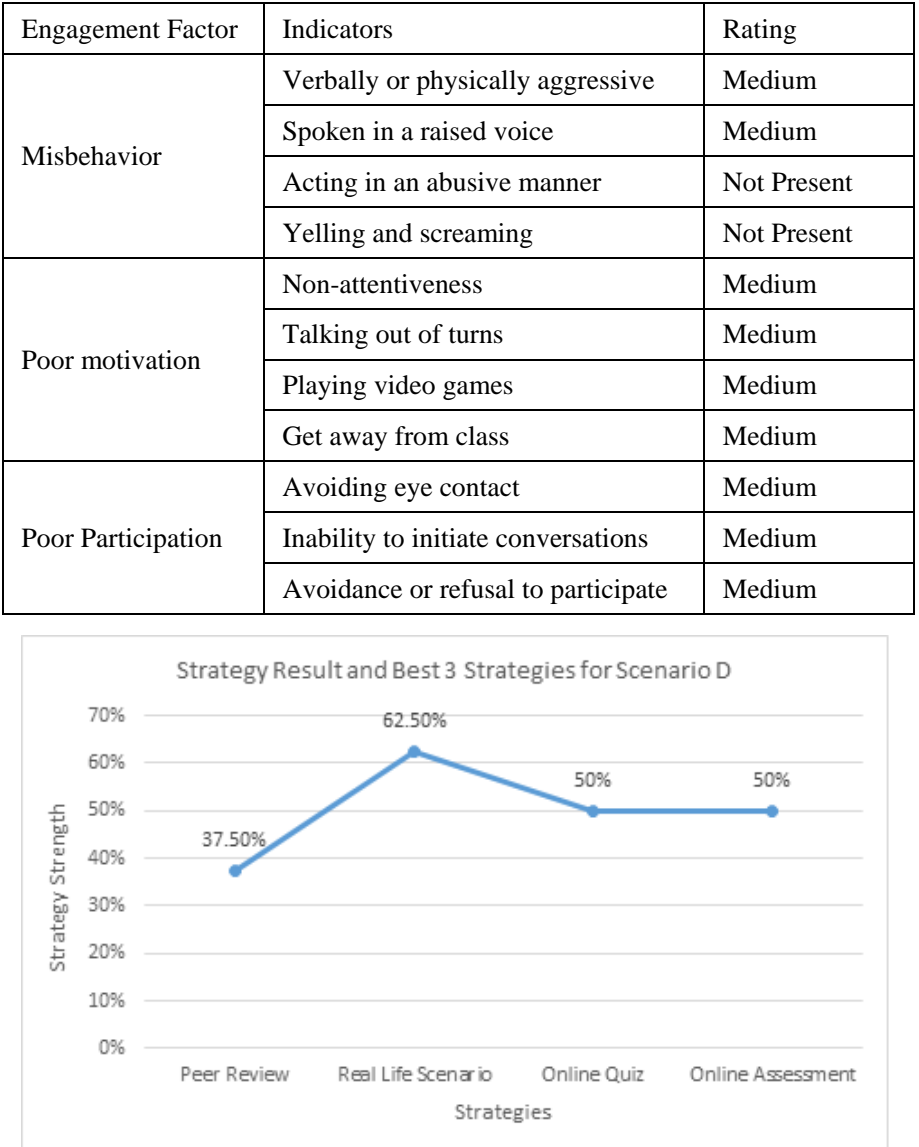

Fig. 12. Selected Strategy Result and Best 3 Strategies for Scenario D.

The Developed tool could provide the improvement to obtain teaching strategy easily compared to a traditional method by using software agents that automatically calculate the strength of each strategy according to environmental settings and also analyses the engagement level and emotional state of students. The values created by the tool include how strategy strength improves engagement and emotional state, reduces humans' time, effort, and cost, and promising in the quality of teaching strategies. The result of the analysis from two different testing includes scenario-based testing and questionnaire feedbacks shows clearly how helpful the classroom simulator tool to the academician and social science researchers. Moreover, the results of the evaluation revealed that the respondents did not experience any difficulty while assessing the tool. The tool is easy to use and not complex as each function is well integrated.

\section{CONCLUSION LiMITATION AND FUTURE WORK}

In this paper, we explored the use of agent-based social simulation and implemented the technology in evaluating the performance of students' engagement in a classroom dynamics simulator. We looked at a typical traditional method used to study the poor student engagement issues for decades which consume considerable time, effort, and very limited settings. Furthermore, since these experiments are applied to humans' society, there are very limited settings to be tested and the costs 
would be excessive if multiple settings are applied. The goal of this study is to automate the student engagement cycle using software agent technology to resolve some of the problems in the process. We demonstrated the use of software agents in measuring the engagement level and identifying the emotional states of students in a classroom dynamic simulator. On the other hand, the agent can calculate teaching strategy strength and recommends the best three strategies to academicians in accordance with their environmental factors.

Our contribution to this research is five-fold. Firstly, we identify three sets of attributes related to this study which are environmental attributes, engagement attributes, and emotional attributes that have been impacting teaching strategies. Then, we developed an agent-based evaluation model where we focus a great deal of our attention on emotional engagement and its associated attributes of existing strategies in enhancing poor students' engagement in classrooms. We thoroughly explored the theories and research findings on students' engagements area. Secondly, we propose a method to evaluate student engagement in a classroom dynamic simulator. We examine the three engagement factors that have been influencing engagement levels in a classroom such as misbehavior, poor motivation, and poor motivation. Thirdly, we examine the emotional factor; anger, anxiety, and boredom derived from engagement factors and study the issues and problems associated with them. The investigation covers the study and analysis of the work process including important aspects that lead our particular attention to which software agent technology could be applied. We propose a technique to identify emotional states that occurs among students' agents. Fourthly, we propose a technique to calculate a teaching strategy in accordance with environmental attributes includes the number of students, class duration and session, type of subjects and year of students, and emotional factors. On top of that, the agents can recommend the best three strategies to be deployed in certain environmental settings. Finally, we develop a solution in the form of a logical model for the classroom dynamic simulator in terms of the overall process flow, measurement of engagement level, analysis of emotional states of students, strategy selection and recommendation, and other novel aspects that could be deployed to improve the poor engagement performance in a classroom. In the proposed simulation, when the lecturer agent, detects low students' engagement, it selects a potential strategy to improve the low students' engagement. Therefore, the lecturer agent is aware of the attributes or specifications of every strategy defined in its knowledge base to decide on the potential one for a particular situation.

While the results of our work in the classroom dynamic simulator show considerable success in the research objectives, there are also some limitations and deficiencies in this work. However, these deficiencies do not compromise the significance of this research. The first limitation, the agent can produce strategy strength selected to particular environmental settings, however, we don't show the impact of strength or how the strategy strength can improve the engagement level due to lack of data. In this case, strategy strength and engagement level, and emotional states of students of the current situation stand as two different components. Another limitation, lecturer emotion is equally important as students' emotions to complete the cycle of the process from the beginning of the selection of strategy, measuring engagement, identifying emotional states of the student, that eventually affect lecturer emotion which triggers them to revise strategy. However, our scope of the study is limited to examine students' emotional state, and we do not develop any formulation to examine or improve lecturer emotions. Another limitation, to determine the engagement level, we use the ordinal scale, nevertheless, a better and more realistic approach could be investigated here such as using fuzzy logic, which will be added to the limitation and future work of this study.

The scope for research in the classroom dynamic simulator overlays the way for many discoveries that could be integrated into agents. We outline here some interesting areas that could be investigated in our future work, (i) Virtual classroom designed with 3D graphical elements to portray emotions transition in colors and propose a technique to show how lecturer emotion being triggered and how it can be used to improve their emotions, (ii) Propose a method to show the interrelation between strategy strength and its ability to improve engagement level in a classroom, (iii) Use real data set from experienced academician on proposing new strategies and show how the improvement of engagement level takes place.

\section{ACKNOWLEDGMENT}

This work is sponsored by Universiti Tenaga Nasional (UNITEN) under the Bold Research Grant Scheme No. J510050002.

\section{REFERENCES}

[1] Owens, D. C., Herman, B. C., Oertli, R. T., Lannin, A. A., \& Sadler, T. D. (2019). Secondary Science and Mathematics Teachers' Environmental Issues Engagement through Socioscientific Reasoning. Eurasia Journal of Mathematics, Science and Technology Education, 15(6), em1693.

[2] Rissanen, A. (2018). Student engagement in large classroom: the effect on grades, attendance and student experiences in an undergraduate biology course. Canadian Journal of Science, Mathematics and Technology Education, 18(2), 136-153.

[3] Inman, C. (2019). Examining Teacher-Student Relationships: Moving from Bullying to Caring (Doctoral dissertation, Southern Illinois University at Edwardsville).

[4] Yazzie-Mintz, E. (2010). Charting the path from engagement to achievement: A report on the 2009 High School Survey of Student Engagement. Bloomington, IN: Center for Evaluation \& Education Policy.

[5] Bature, I. J. (2020). The Mathematics Teachers Shift from the Traditional Teacher-Centred Classroom to a More Constructivist Student-Centred Epistemology. Open Access Library Journal, 7(5), 126.

[6] Thang, S. M. (2009). Investigating autonomy of Malaysian ESL learners: A comparison between public and private universities. 3L; Language, Linguistics and Literature, The Southeast Asian Journal of English Language Studies., 15, 97-124.

[7] Cooper, K. M., Krieg, A., \& Brownell, S. E. (2018). Who perceives they are smarter? Exploring the influence of student characteristics on student academic self-concept in physiology. Advances in physiology education, 42(2), 200-208.

[8] Dasari, B. (2009). Hong Kong students' approaches to learning: crosscultural comparisons. US-China Education Review, 6(12), 46-58.

[9] Subramainan, L., \& Mahmoud, M. A. (2020). A Systematic Review on Students' Engagement in Classroom: Indicators, Challenges and 
Computational Techniques. International Journal of Advanced Computer Science and Applications, 11(1), 105-115.

[10] PISA, O. (2012). Results in Focus: What 15-year-olds know and what they can do with what they know. 2014-12-03]. http:////www, oecd. org/pisa,/keyfindings,/pisa-2012-results-overview, pdf.

[11] Yu, R., \& Singh, K. (2018). Teacher support, instructional practices, student motivation, and mathematics achievement in high school. The Journal of Educational Research, 111(1), 81-94.

[12] Pryor, J. H., Hurtado, S., DeAngelo, L. E., Blake, L. P., \& Tran, S. (2010). The American freshman: National norms fall 2009. Univ of California Press.

[13] Mann, S., \& Robinson, A. (2009). Boredom in the lecture theatre: An investigation into the contributors, moderators, and outcomes of boredom among university students. British Educational Research Journal, 35(2), 243-258.

[14] Nikish. C, (2013) Hobsons (http://www.hobsons.com)student engagement (http://www.naviance.com/blog/c/studentengagemeNT).

[15] Teoh, H. C., Abdullah, M. C., Roslan, S., \& Daud, S. (2013). An investigation of student engagement in a Malaysian Public University. Procedia-Social and Behavioral Sciences, 90, 142-151.

[16] Macal, C. M., \& North, M. J. (2009, December). Agent-based modeling and simulation. In Winter simulation conference (pp. 86-98). Winter Simulation Conference.

[17] Arechar, A. A., Gächter, S., \& Molleman, L. (2018). Conducting interactive experiments online. Experimental economics, 21(1), 99-131.

[18] Lei, H., Cui, Y., \& Zhou, W. (2018). Relationships between student engagement and academic achievement: A meta-analysis. Social Behavior and Personality: an international journal, 46(3), 517-528.

[19] Zhang, Z. V., \& Hyland, K. (2018). Student engagement with teacher and automated feedback on L2 writing. Assessing Writing, 36, 90-102.

[20] Pekrun, R., Cusack, A., Murayama, K., Elliot, A. J., \& Thomas, K. (2014). The power of anticipated feedback: Effects on students' achievement goals and achievement emotions. Learning and Instruction, $29,115-124$.

[21] Sharp, J. G., Sharp, J. C., \& Young, E. (2020). Academic boredom, engagement and the achievement of undergraduate students at university: A review and synthesis of relevant literature. Research Papers in Education, 35(2), 144-184.

[22] Lamborn, S., Newmann, F., \& Wehlage, G. (1992). The significance and sources of student engagement. Student engagement and achievement in American secondary schools, 11-39.

[23] Parsons, J., \& Taylor, L. (2011). Improving student engagement. Current issues in education, 14(1).

[24] Fredricks, J. A., \& McColskey, W. (2012). The measurement of student engagement: A comparative analysis of various methods and student self-report instruments. In Handbook of research on student engagement (pp. 763-782). Springer US.

[25] Hogan, R., Hogan, J., \& Warrenfeltz, R. (2007). The Hogan guide: Interpretation and use of Hogan inventories. Hogan Assessment Systems.
[26] Chin, K. O., Gan, K. S., Alfred, R., Anthony, P., \& Lukose, D. (2014). Agent architecture: An overviews. Transactions on science and technology, 1(1), 18-35.

[27] Jassim, O. A., Mahmoud, M. A., \& Ahmad, M. S. (2015). A multi-agent framework for research supervision management. In Distributed Computing and Artificial Intelligence, 12th International Conference (pp. 129-136). Springer, Cham.

[28] Mahmoud, M. A., \& Ahmad, M. S. (2016, August). A prototype for context identification of scientific papers via agent-based text mining. In 2016 2nd International Symposium on Agent, Multi-Agent Systems and Robotics (ISAMSR) (pp. 40-44). IEEE.

[29] Mahmoud, M. A., \& Ahmad, M. S. (2015, August). A self-adaptive customer-oriented framework for intelligent strategic marketing: A multi-agent system approach to website development for learning institutions. In 2015 International Symposium on Agents, Multi-Agent Systems and Robotics (ISAMSR) (pp. 1-5). IEEE.

[30] Salleh, A. M., Desa, M. M., \& Tuit, R. M. (2013). The Relationship between the Learning Ecology System and Students' Engagement: A Case Study in Selangor. Asian Social Science, 9(12), 110.

[31] Subramainan, L., \& Mahmoud, M. A. (2020, August). Academic Emotions Review: Types, Triggers, Reactions, and Computational Models. In 2020 8th International Conference on Information Technology and Multimedia (ICIMU) (pp. 223-230). IEEE.

[32] Subramainan, L., Mahmoud, M. A., Ahmad, M. S., \& Yusoff, M. Z. M. (2017, June). A simulator's specifications for studying students' engagement in a classroom. In International Symposium on Distributed Computing and Artificial Intelligence (pp. 206-214). Springer, Cham.

[33] Subramainan, L., Mahmoud, M. A., Ahmad, M. S., \& Yusoff, M. Z. M. (2016, August). A conceptual emotion-based model to improve students engagement in a classroom using agent-based social simulation. In 2016 4th International Conference on User Science and Engineering (iUSEr) (pp. 149-154). IEEE.

[34] Subramainan, L., Mahmoud, M. A., Ahmad, M. S., \& Yusoff, M. Z. M. (2016, August). Evaluating students engagement in classrooms using agent-based social simulation. In 2016 2nd International Symposium on Agent, Multi-Agent Systems and Robotics (ISAMSR) (pp. 34-39). IEEE.

[35] Subramainan, L., Mahmoud, M. A., Ahmad, M. S., \& Yusoff, M. Z. M. (2016). An Emotion-based Model for Improving Students' Engagement using Agent-based Social Simulator. International Journal on Advanced Science, Engineering and Information Technology, 6(6), 952-958.

[36] Subramainan, L., Yusoff, M. Z. M., \& Mahmoud, M. A. (2015, August). A classification of emotions study in software agent and robotics applications research. In 2015 International Symposium on Agents, Multi-Agent Systems and Robotics (ISAMSR) (pp. 41-46). IEEE.

[37] Subramainan, L., Yusoff, M. Z. M., \& Mahmoud, M. A. (2015, August). A classification of emotions study in software agent and robotics applications research. In 2015 International Symposium on Agents, Multi-Agent Systems and Robotics (ISAMSR) (pp. 41-46). IEEE. 